\title{
NEW PSA PROCESS WITH INTERMEDIATE FEED INLET POSITION OPERATED WITH DUAL REFLUXES: APPLICATION TO CARBON DIOXIDE REMOVAL AND ENRICHMENT
}

\author{
DOUDOU DIAGNE \\ Graduate School of Science and Technology, Kumamoto University \\ MOTONOBU GOTO AND TsUTOMU HIROSE* \\ Department of Applied Chemistry, Kumamoto University, \\ Kurokami 2-39-1, Kumamoto 860
}

Key Words: Adsorption, Pressure Swing Adsorption, Gas Separation, Carbon Dioxide, Dual Reflux, Stripping, Enriching

\begin{abstract}
A new PSA process was deyeloped and applied to the removal and enrichment of carbon dioxide from air$\mathrm{CO}_{2}$ mixtures. This PSA process is constituted by a dual reflux policy and admission of the feed gas mixture at an intermediate axial position of the column. The influences of the feed inlet position and reflux ratio on process performance were experimentally investigated. For both parameters there is respectively an optimum value at which the process performance was relatively higher. At the optimal values it was possible to concentrate and remove the $\mathrm{CO}_{2}$ simultaneously beyond a factor of the pressure ratio $P_{a} / P_{d}$, a situation unattainable in the conventional PSA in which the feed gas is supplied at the end of the column.
\end{abstract}

\section{Introduction}

Pressure swing adsorption, abbreviated PSA, is a cyclic separation process for gas mixtures. Since its invention concurrently by Skarstrom ${ }^{7)}$ and GuerrinDomine ${ }^{1)}$, PSA technology has gained growing interest for the last thirty years owing to its low energy requirements and low capital investment costs. The most popular industrial applications of PSA technology initially were the drying of air and the purification of hydrogen. Later, applications to bulk separations such as oxygen and nitrogen production were also developed.

At equilibrium, the partial pressure of the adsorbed solute is equal to that of the contacting gas phase. Therefore, by swinging the total pressure between a low and a high value, the solute is readily removed from the solid adsorbent, which becomes regenerated. This operation is repeated according to a programmed sequence. Operating policies are involved but the most important consideration is that a minimum difference between adsorption and desorption pressure must exist to separate a mixture to a given degree.

A new PSA process with intermediate feed inlet position is proposed in this study and the performance is tested experimentally by application to the simultaneous removal and enrichment of $\mathrm{CO}_{2}$. The main objective of the study is to show the advantage that the minimum difference of pressure can be decreased in the new process compared with the process with end-feed position. Another objective is to show the existence of optimal values of the feed inlet position and reflux ratio.

\footnotetext{
* Received July 23, 1993. Correspondence concerning this article should be addressed to T. Hirose.
}

\section{Principle of PSA with Intermediate Feed Inlet Position}

Generally, in PSA operations the objective is either to remove or to concentrate one or many components from a binary or multicomponent feed gas mixture. Conventional PSA schemes for the purposes of removal (stripping) and concentration (enriching) are respectively illustrated in Fig. 1 and Fig. 2. Each figure shows a situation during a half-cycle in which the left column is subjected to high pressure. The configuration during the next half-cycle corresponds to a view from the opposite side of the paper.

In Fig. 1, a part of the product gas from the highpressure column is purged to the low-pressure column as a "stripping" reflux. The mole fraction $\left(X_{L}\right)$ of the adsorbate in the lean gas from the bottom of the high-pressure column can be lowered to a value nearly equal to zero $\left(X_{L} \rightarrow 0\right)$. However, the mole fraction $\left(X_{E}\right)$ of the adsorbate at the top of the low-pressure column cannot be increased at the same time up to a value higher than the pressure ratio $\left(P_{a} / P_{d}\right)$ resulting in an enriching limitation. Fig. 2 is appropriate for concentration or enrichment. In this configuration a part of the product gas from the right column is recycled to the left column after compression as an "enriching" reflux. The solute in the feed gas can be concentrated up to a very high concentration $\left(X_{E} \rightarrow 1\right)$. However,the mole fraction at the bottom of the left column represented by $X_{L}$ cannot be lowered below a factor $P_{d} / P_{a}$ resulting in a stripping limitation.

The above two limitations can be overcome by reprocessing enriched gas leaving the stripping PSA in the enriching PSA, and lean gas leaving the enriching 


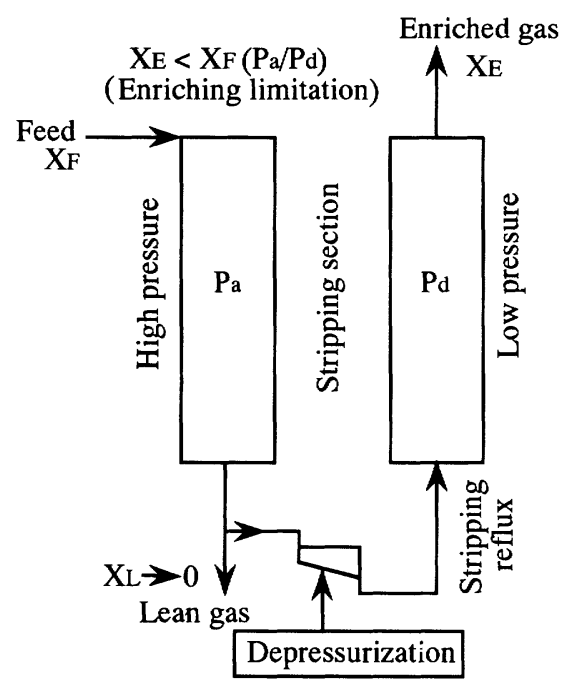

Fig. 1 PSA operation for stripping case

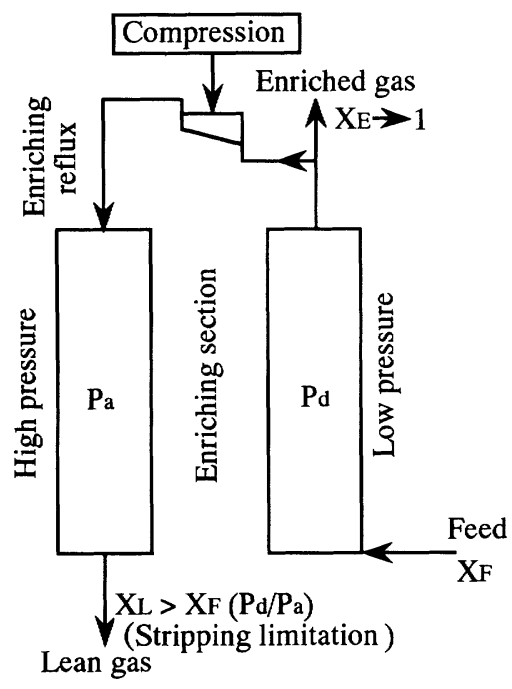

Fig. 2 PSA operation for enrichment case

PSA in the stripping PSA. This concept leads to a new PSA process ${ }^{2)}$ of which a conceptual diagram is presented in Fig. 3. It is a combination of the two conventional PSA units represented by Figs. 1 and 2 and based on a dual reflux policy. The feed gas mixture is supplied at an intermediate position of one single column under high or low pressure.

Fig. 3 shows the case of low-pressure feed in our process. A part of the enriched gas leaving the top of the right column is recycled at the top of the left column, resulting in an "enriching" reflux ratio between the recycled enriched gas flow rate $\left(R_{E}\right)$ and the enriched gas product flow rate $\left(Q_{E}\right)$. On the other hand, a part of the lean gas leaving the bottom of the left column is also recycled at the bottom of the right column, resulting in a "stripping" reflux ratio between the recycled lean gas flow rate $\left(R_{L}\right)$ and the lean gas product flow rate $\left(Q_{L}\right)$. The case of high-pressure feed may be visualized similarly. By analogy to distillation, the feed inlet position divides each column into enriching and stripping sec-

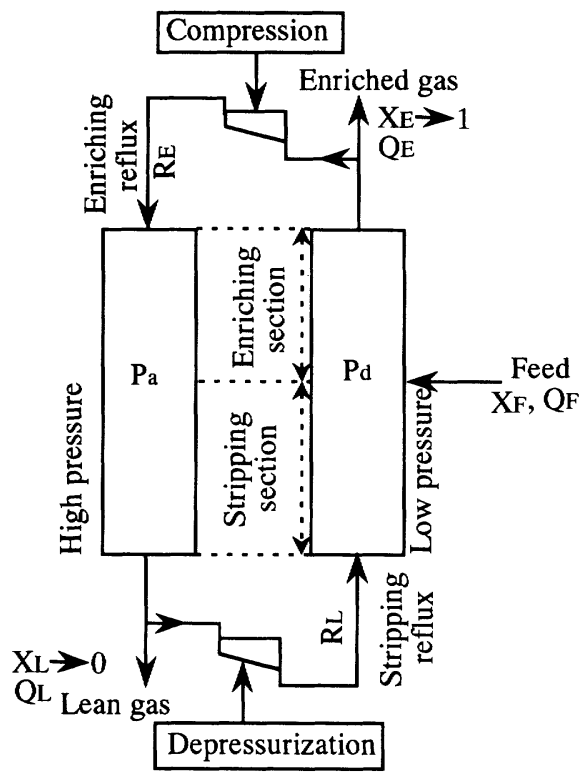

Fig. 3 Conceptual diagram of PSA with intermediate feed inlet position

tions, both at the same pressure. The feed inlet position and reflux ratio are important operating variables, and for an appropriate combination of them this PSA process can especially give two types of high-purity productsboth enriched gas and lean gas.

The concept of the dual reflux policy and intermediate feed inlet position in PSA process has been employed in the simultaneous production of high-purity nitrogen and oxygen ${ }^{5)}, \mathrm{CO}_{2}$ enrichment with high recovery ${ }^{3)}$, ultra-high purification of hydrogen ${ }^{6)}$ and so on. However, the operating policy was quite different compared to our process. Each column or section of their processes undergoes the same sequence of a common permutation while the enriching section and the stripping section are in different permutations in the present process. Each column should be of the same size in the former but the size of each section can be designed independently in the latter. In other words, the former is a symmetric single-stage unit and the latter an asymmetric two stages - in - series unit.

Another type of enriching reflux can be seen in multistaged enrichment of propylene ${ }^{4)}$. However, it is interpreted as a multistage crossflow unit of stripping PSA and is different to the present one. Thus the present type of PSA is not found in the literature within the authors' knowledge.

\section{Experimental}

The performance of the PSA process was investigated for the adsorption of carbon dioxide at various mole fractions. The experimental apparatus of PSA with intermediate feed inlet position is shown in Fig. 4. It consisted of two PSA-columns made of brass. Each column had a diameter of $18 \mathrm{~mm}$ and a total length of 


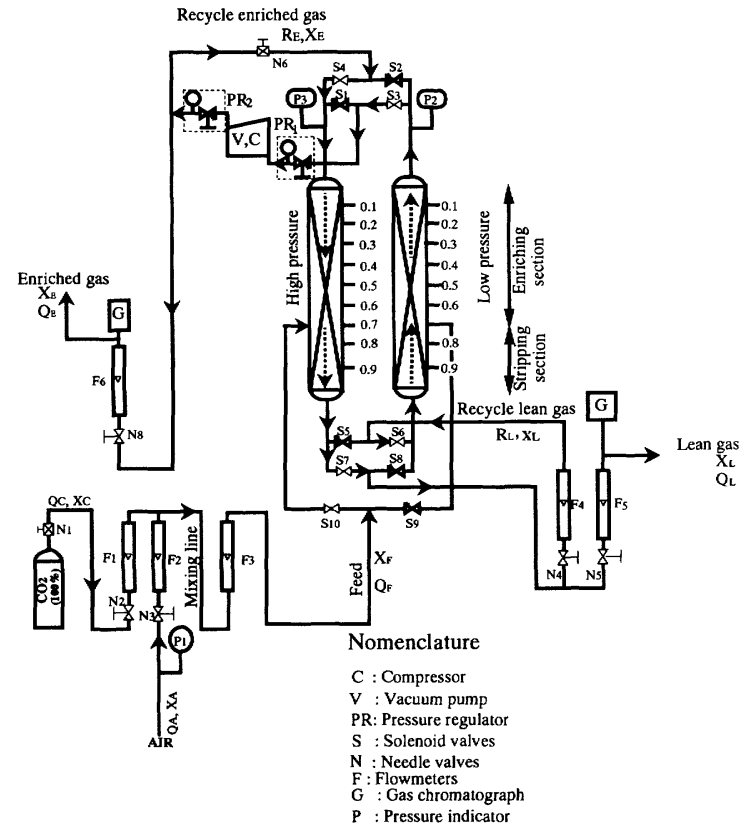

Fig. 4 Experimental apparatus

$1000 \mathrm{~mm}$ and was equipped with nine feed entrance possibilities. Zeolite MS $13 \mathrm{X}$ was used as adsorbent and a short half-cycle time was fixed at $2 \mathrm{~min}$ or $4 \mathrm{~min}$. Both cases of high-pressure feed and low-pressure feed were considered. Referring to Fig. 4, the process configuration for high-pressure feed consisted of adsorption through the left (high-pressure) column of the amount of feed + recycled enriched gas, both at atmospheric pressure; and desorption through the right (low-pressure) column by admission of the recycled lean gas in addition to an evacuation by means of a vacuum pump. In the case of low-pressure feed, it consisted of desorption through the low-pressure column by the feed + recycled lean gas at atmospheric pressure. Adsorption of recycled enriched gas through the high-pressure column was carried out by means of a compressor.

\section{Results and Discussion}

\subsection{Feasibility of carbon dioxide removal and enrichment}

The feasibility of simultaneous removal and enrichment of carbon dioxide by means of this PSA process was first investigated. For this purpose, the mole fraction of carbon dioxide in the feed gas $\left(X_{F}\right)$ was varied between $0-20 \%$, keeping the other paramaters constant. The results for the high-pressure feed are presented in Fig. 5. The left side and the right side of the ordinate refer respectively to the enrichment ratio of carbon dioxide in the enriched-gas product $\left(X_{E} / X_{F}\right)$ and the reduction ratio of carbon dioxide in the lean-gas product $\left(X_{L} / X_{F}\right)$.

As a consequence, it is noticeable that the enrichment ratio $\left(X_{E} / X_{F}\right)$ was never less than 4.4 and the reduction ratio $\left(X_{L} / X_{F}\right)$ never greater than 0.15 . There-

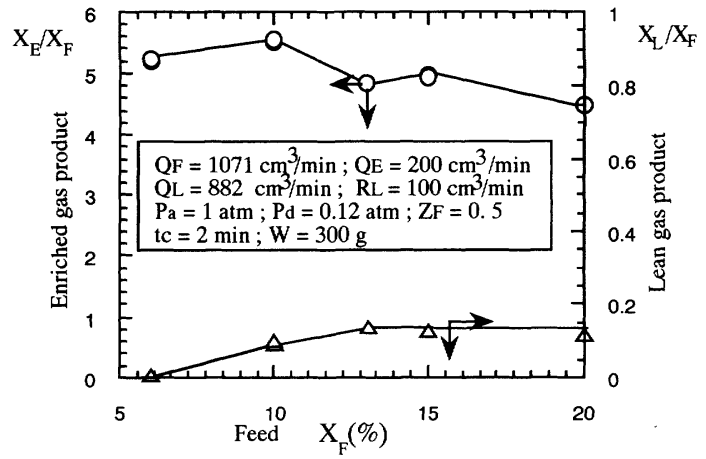

Fig. 5 Enrichment and reduction ratio as a function of feed gas mole fraction (high-pressure feed)

fore from a feed gas mixture containing $20 \%$ of carbon dioxide, the carbon dioxide mole fraction in the enriched gas stream was around $90 \%$ and that in the lean gas was around $2.5 \%$, showing good ability for this PSA process to concentrate and simultaneously remove carbon dioxide.

\subsection{Effects of the feed inlet position}

Since one of the characteristics of this new PSA process is relative to feeding at an intermediate position of the column,the influence of the feed inlet position on the process performance was investigated. Therefore,the feed gas was supplied from a different feed inlet position while the gas flow rates, the feed concentration, the amount of adsorbent and the pressure ratio were kept constant. Ambient air containing 350-450 ppm of $\mathrm{CO}_{2}$ was used as feed gas and the experiment was carried out for the low-pressure feed. The results are shown in Fig. 6. In this figure the abscissa refers to the feed inlet position or the dimensionless length of the enriching section $\left(Z_{F}\right)$ defined as: $Z_{F}=L_{R} /\left(L_{R}+L_{S}\right)$.

It can be seen from Fig. 6 that when $Z_{F}$ is very small or short it is quite impossible to remove and concentrate successfully the carbon dioxide contained in the feed gas. On the other hand, when $Z_{F}$ is very high or long, a noticeable decrease of the mole fraction of carbon dioxide in the enriched gas is observed. Therefore, as indicated in Fig. 6, there is an optimum feed inlet position at which the process performance was higher. This optimum feed inlet position was equal to $Z_{F}=0.5$ in this condition, which corresponds to both an enriching and a stripping section length $L_{R}=L_{S}=500 \mathrm{~mm}$. At this feed position, the enrichment ratio of carbon dioxide in the enriched gas was the highest $\left(X_{E} / X_{F}=6.5\right)$ and the reduction ratio in the lean gas streams was the lowest $\left(X_{L} / X_{F}=0.15\right)$. Thus the feed gas was enhanced and reduced simultaneously by a factor of $6-7$, which is much higher than the pressure ratio $\left(P_{a} / P_{d}\right)=2$.

As mentioned in the preceding section, the conventional PSA with end-feed position has a thermodynamic limitation as a result of the enriching or stripping limitation, by which the feed gas cannot be enriched or reduced simultaneously beyond a factor of the pressure ratio. In other words, a minimum pressure is required in the conventional PSA to attain a given degree of simul- 

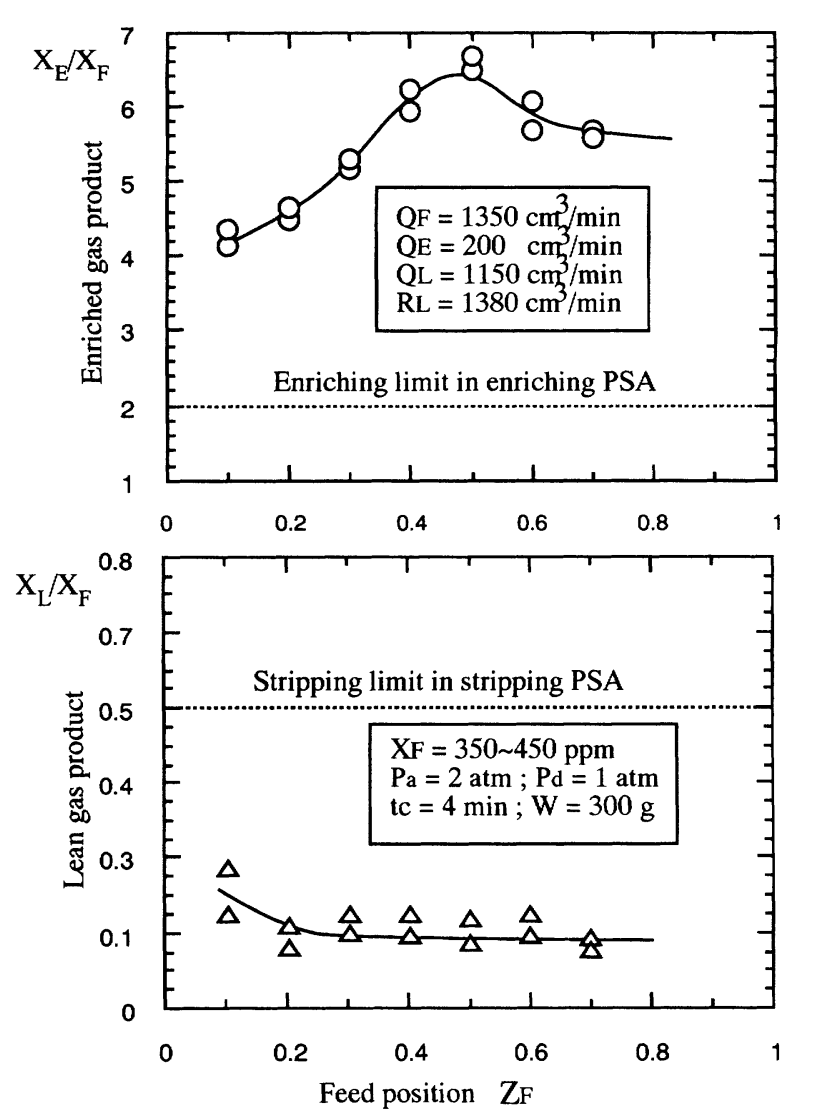

Fig. 6 Enrichment and reduction ratio as a function of the feed inlet position (low-pressure feed)

taneous enrichment and reduction. The recycled enriched gas must be compressed up to 6-7 atm, at least in the conventional enrichment PSA, to obtain the same separation performance as in the present case. It should be noted that in the proposed PSA process, high performance can be attained at a pressure ratio as low as 2 since it is free from the thermodynamic limitation.

\subsection{Effects of the reflux ratio}

Since this PSA process is also characterized by a dual reflux policy, it is interesting to investigate the influence of the reflux ratio on the product concentration. The variation of one reflux ratio such as the "stripping" reflux ratio $\left(R_{L} / Q_{L}\right)$ is enough to interpret the resulting influence on the product concentration since the enriching reflux ratio $\left(R_{E} / Q_{E}\right)$ is related to $R_{L} / Q_{L}$ by:

$$
\frac{R_{E}}{Q_{E}}=\left(1+\frac{R_{L}}{Q_{L}}\right)\left(\frac{Q_{L}}{Q_{E}}\right)
$$

from the viewpoint of material balance. Therefore,the recycled lean gas $R_{L}$ was varied, keeping constant the other parameters. The "stripping" reflux ratio was then calculated and its influence on the enrichment and reduction ratios was investigated. The mole fraction of $\mathrm{CO}_{2}$ in the feed was adjusted to $20 \%$ and the experiment was carried out for the high-pressure feed.

The results are shown in Fig. 7. When the reflux ratio is small $\left(R_{L} / Q_{L}=0.1\right)$ the enrichment ratio $\left(X_{E} / X_{F}\right)$ is low and the reduction ratio $\left(X_{L} / X_{F}\right)$ high, resulting in
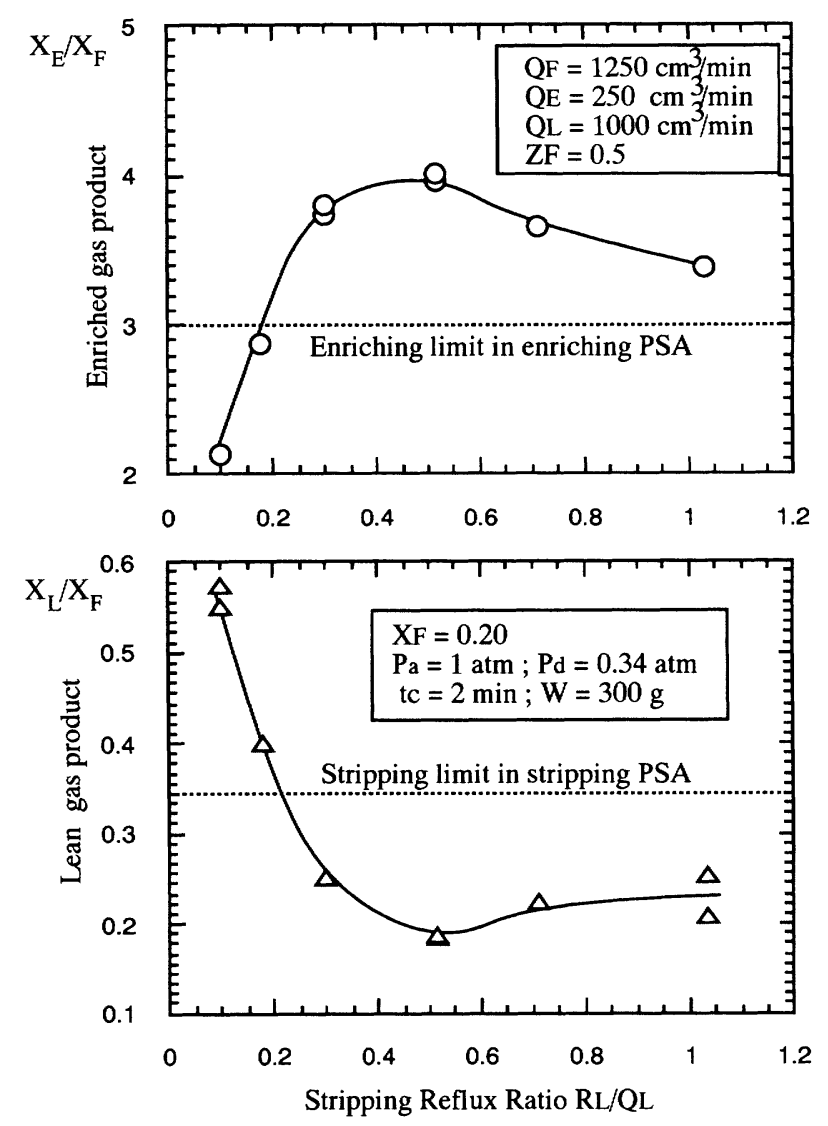

Fig. 7 Influence of the "stripping" reflux ratio on the enrichment and reduction ratio (high-pressure feed)

quite low performance. On the other hand, when the reflux ratio increases, the process performance improves and there is an increase of the enrichment ratio and a decrease of the reduction ratio. However, when the reflux ratio tends to high values $\left(R_{L} / Q_{L} \geq 0.6\right)$ the residence time of the gas inside the column becomes shorter, resulting in a decrease in performance. Therefore, there is an optimum reflux ratio at which process performance was higher. Especially at the optimum reflux ratio $(0.4 \leq$ $\left.R_{L} / Q_{L} \leq 0.5\right)$, it was possible to concentrate the carbon dioxide contained in the feed gas at a ratio $\left(X_{E} / X_{F}\right)=4$ higher than the pressure ratio $P_{a} / P_{d}=2.9$ while the reduction ratio $\left(X_{L} / X_{F}\right)$ was around 0.19 . In other words, since the feed gas consisted of $20 \%$ of carbon dioxide, the mole fraction of carbon dioxide in the enriched gas and lean gas streams was respectively $80 \%$ and $3.8 \%$, corresponding to a fractional recovery of $80 \%$. These values of the enrichment and reduction ratio are again better than that for the end-feed PSA processes controlled by the pressure ratio.

Thus the proposed PSA process has potential applicability to $\mathrm{CO}_{2}$ fixation from flue gas, particularly in the case where both enriched gas and lean gas products are not necessarily required at high pressure.

One advantage of this PSA process is that the difference of pressure or the pressure ratio required to attain a certain degree of product purity can be sensibly 
reduced. Basically, since the power consumption of the compressor or the vacuum pump is given by the product of the gas flow rate and value of the pressure difference, the energy requirement becomes lower, resulting in principle in a lower separation cost. On the contrary, it should be noted that at low pressure difference or ratio, the gas flow rate must be increased to keep also a certain degree of product purity. At a low pressure ratio an increase in flow rate tends to increase the power consumption of the compressor or the vacuum pump. Therefore, in the point of view of energy saving, further investigations are necessary to estimate the power consumption for a given throughput ratio and reflux ratio to yield a certain degree of enrichment and reduction and to gain a better understanding of this PSA process towards a rigorous optimization study.

\section{Conclusions}

PSA with intermediate feed inlet position shows good ability for simultaneous removal and enrichment of carbon dioxide from both dense and dilute systems. The above experimental results can be considered as the doorway to a potential application of this process to the production of oxygen or nitrogen, methane purification from biogases and the like.

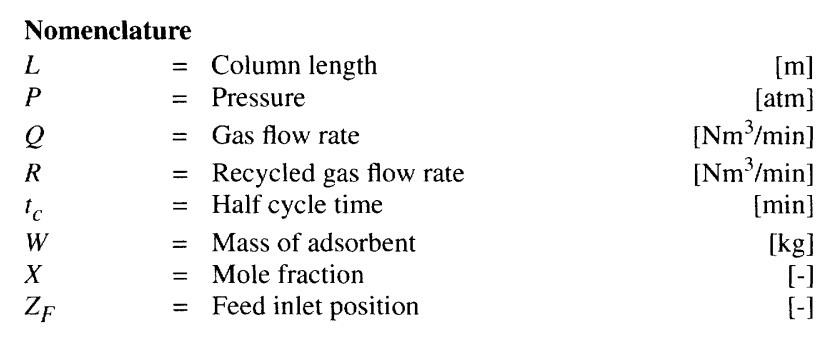

$<$ Subscripts $>$

$$
\begin{array}{ll}
a & =\text { Adsorption } \\
d & =\text { Desorption } \\
E & =\text { Enriched gas } \\
F & =\text { Feed gas } \\
L & =\text { Lean gas } \\
R & =\text { Rectifying section } \\
S & =\text { Stripping section }
\end{array}
$$

Literature Cited

1) Guerrin de Montgareuil, P. and D. Domine: U.S. Patent $3,155,468$ (1964)

2) Hirose, T., 2nd China-Japan-USA symposium on adsorption, p. 123 (1991)

3) Kanemaru, T., Ch. Nishino, Ch. Eng. (in Japanese), 9,. 66-71 (1990)

4) Mersmann, A., U. Munstermann and J. Schadl, Chem. Ing. Tech., 55, No. 6,. 446-458 (1983)

5) Mikami, K., S. Ikumi and S. Ibaraki, Mitsui Zosen Giho (in Japanese), 137, 44-49 (1989)

6) Sircar, S., Sep. Sc. Tech., 25 (11 and 12), 1087-1099 (1990)

7) Skarstrom, C.W.: U.S. Patent 2,944,627 (1960)

(Parts of this work were presented at the annual and the autumn meetings of the Society of Chemical Engineers of Japan, respectively, in April 1992 at Osaka and in October 1992 at Tokyo.) 